\title{
Synapse to Circuit - Correlative Microscopy Workflows for Functional Analysis of the Brain
}

\author{
Naomi Kamasawa ${ }^{1 *}$, Connon I. Thomas ${ }^{1}$, Melissa A. Ryan ${ }^{1}$ and Debbie Guerrero-Given ${ }^{1}$ \\ 1. Electron Microscopy Core Facility, Max Planck Florida Institute for Neuroscience, Jupiter, FL, USA. \\ * Corresponding author: naomi.kamasawa@mpfi.org
}

In brain networks, neurons are interconnected via numerous synapses which transmit information for computations of the cells. Neuronal activity can be recorded with light microscopy (LM) techniques, however, resolution of electron microscopy (EM) is required to analyze the synaptic ultrastructure. By correlating LM and EM data, a more complete understanding of neuronal computation is achieved, and recent advancements in scanning EM (SEM) have made this volumetric correlation more attainable. This paper will discuss two workflows that we established for functional LM and EM correlation using SEM; 1) viral vector-driven expression of a dual microscopic marker to identify labeled profiles under both LM and EM in the brain (Fig. 1); 2) correlation of two-photon in vivo LM and volumetric EM at the synapse level using native fiducial markers with no labeling (Fig. 2).

First, to identify specific cell types under both LM and EM, we developed a helper-dependent adenoviral (HdAd) vector harboring EGFP and a modified ascorbate peroxidase targeted to the mitochondria matrix (mito-APEX2), as we reported last year [1]. Chemical, molecular, or genetic perturbation can be used on particular synaptic proteins with the goal of understanding synaptic function. Because the packaging size of the HdAd allows for labeling and genetic modification, the dual marker is a powerful tool to correlate physiological and morphological changes following this perturbation. We first used the expressed GFP as a marker for electrophysiology (Fig. 1A), then confirmed electron densely labeled mitochondria with transmission EM (TEM) (Fig.1B). We further applied high-throughput EM methods - serial section SEM array tomography (sSSEM) and serial block face SEM (SBF-SEM) - for volumetric morphological analysis (Figs. 1C-E). The ssSEM was suitable to analyze fine structure of synapses due to high electron dosage per pixel. However, serial section-based 3D reconstruction had unavoidable image alignment issues. SBF-SEM was ideal for reconstructing large volumes to investigate the morphology of cells. We found optimization of the heavy metal staining protocol together with the improved sensitivity of the OnPoint BSE detector (Gatan) enabled the differentiation of APEX2 labeled electron dense mitochondria from others in SBF-SEM imaging within reasonable total volume imaging time.

In our second workflow, we sought to correlate EM volume data and in vivo two-photon microscopy, which has ability to capture neuronal activity in the whole brain (Fig. 2A). After live imaging, the brain slices were prepared for volume EM acquisition (Fig. 2B). We further optimized the 3D volume EM sample preparation protocol [2] to fit to our samples to visualize clear synaptic structure while holding enough electron conductivity into a certain depth of the tissue to capture the dendritic tree of the neuron that was imaged by the two-photon microscope before. We used blood vessels as fiducial markers to correlate two-photon calcium imaging and SBF-SEM (Fig. 2C). In the SBF-SEM imaging, the Focal Charge Compensation module (FCC, ZEISS) significantly improved image quality and reduced beam damage of the sample. Furthermore, we introduced a virtual reality visualization system (syGlass, IstoVisio) to overlay the SBF-SEM data and two-photon/confocal microscope images. The collected data sets provided the structural characteristics of synapses to understand how synaptic strength affects 
functional computation in the single neuron (Fig. 2D), and accumulation of these data sets will lead us to have better understanding of basic properties neural circuits [3].

\section{References:}

[1] C I Thomas et al., Microscopy \& Microanalysis 24(S1), (2018), p. 1352.

[2] Y Hua, P Laserstein and M Helmstaedter, Nat. Commun. 6 (2015), p. 7923.

[3] The authors thank the Applications and Services of Carl Zeiss Microscopy and IstoVisio for their support.
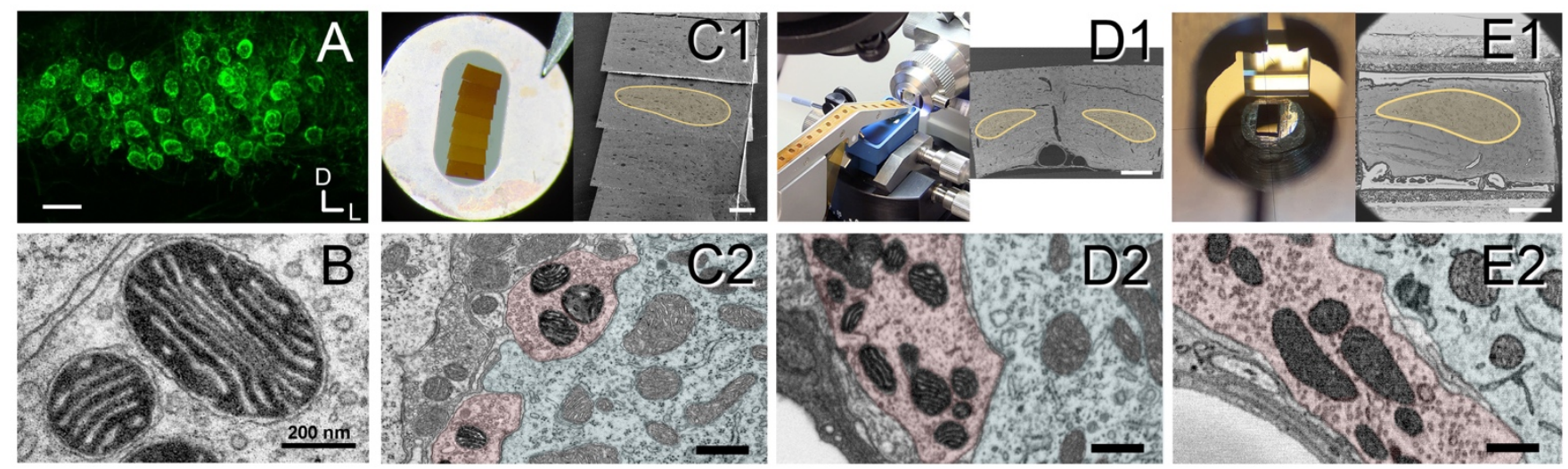

Figure 1. EGFP-mitoAPEX2 labeling in different microscopic platforms. (A) EGFP fluorescence can be seen in calyx of Held terminals. Bar $=1 \mu \mathrm{m},(\mathrm{B})$ High magnification TEM image of APEX2 labeled mitochondria. (C-E) Comparisons of different EM techniques (C, TEM; D, ssSEM; E, SBF-SEM). Bars $=100 \mathrm{~nm}$ for $\mathrm{C} 1-\mathrm{E} 1,500 \mathrm{~nm}$ for $\mathrm{C} 2-\mathrm{E} 2$. APEX2 positive mitochondria were differentiable in all methods.
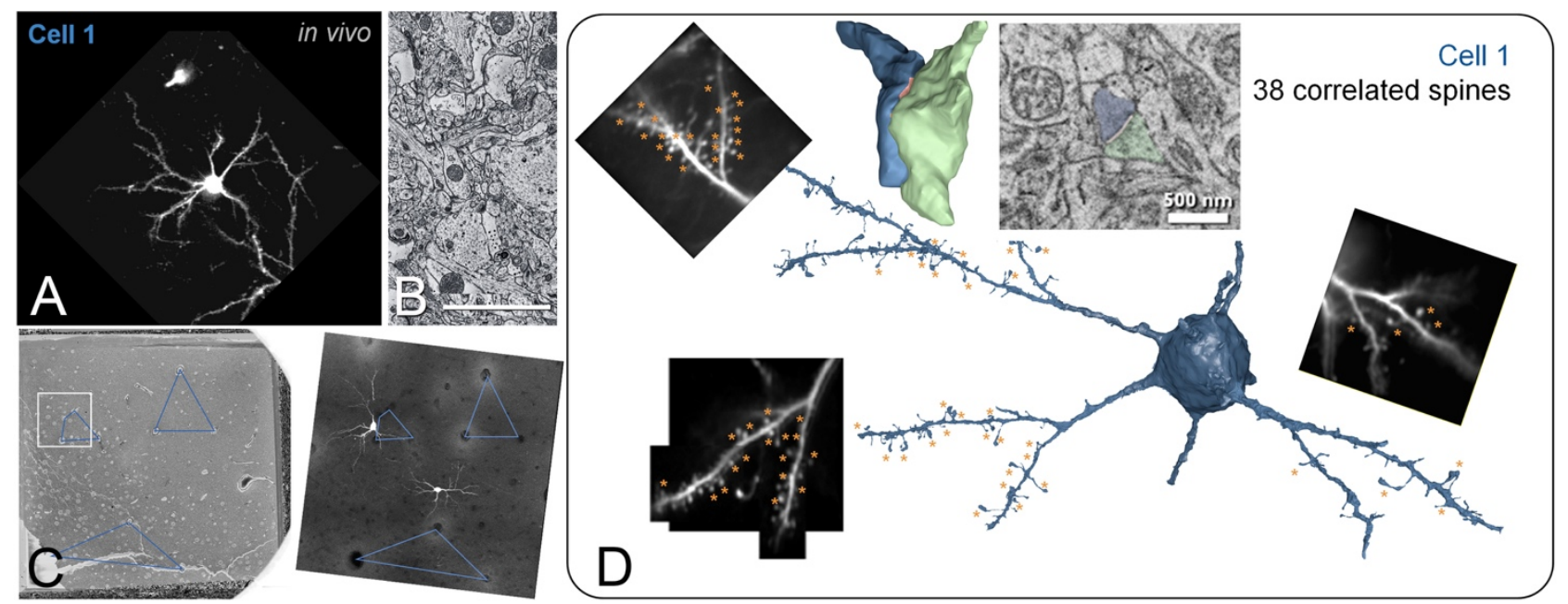

Figure 2. Example of correlative imaging of a cell from two-photon in vivo imaging to SEM. (A) Twophoton in vivo calcium image. (B) SBF-SEM image. (C) A scheme how to define the position of the cell imaged with LM in a block face. Note the triangles among blood vessels as markers. (D) A summary showing spines on three branches of dendrites correlated between two-photon images and EM, then 3D reconstructed from serial images. 